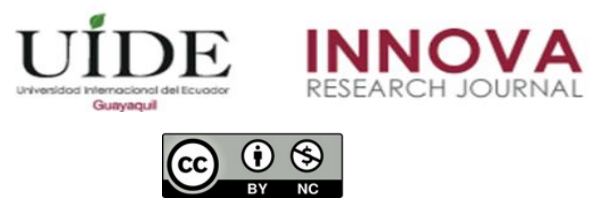

INNOVA Research Journal, ISSN 2477-9024

(Septiembre-Diciembre 2019). Vol. 4, No.3.1 pp. 55-71

DOI: https://doi.org/10.33890/innova.v4.n3.1.2019.975

URL: http://revistas.uide.edu.ec/index.php/innova/index

Correo: innova@uide.edu.ec

\title{
TIC para la inclusión educativa universitaria en la UPS Sede Guayaquil
}

\section{ICT for university educational inclusion in the UPS headquarter Guayaquil}

\author{
Bertha Alice Naranjo Sánchez \\ https://orcid.org/0000-0002-4386-2335 \\ Kléver Andrés Chávez Huacón \\ https://orcid.org/0000-0001-5781-4898 \\ Universidad Politécnica Salesiana, Ecuador
}

Autor para correspondencia: bnaranjo@ups.edu.ec; kchavez@est.ups.edu.ec

Fecha de recepción: 21 de marzo de 2019 - Fecha de aceptación: 15 de octubre de 2019

\section{Resumen}

La inclusión educativa universitaria es un tema que ha sido muy poco investigado en nuestro país. Muchas variables inciden en una plena inclusión educativa de estudiantes con discapacidad a nivel superior. El objetivo de este artículo es identificar las TIC que los estudiantes de diferentes carreras de la Universidad Politécnica Salesiana Sede Guayaquil utilizan para favorecer su inclusión, así como el porcentaje de uso de las TIC que ofrece el Centro de Apoyo para la Inclusión de la UPS. A través de la revisión bibliográfica y el análisis documental, entrevistas a personal administrativo, técnico y docente de la universidad, así como de encuestas aplicadas a estudiantes con discapacidad se pudo obtener información relevante sobre las TIC que se utilizan en el proceso de enseñanza aprendizaje inclusivo. Como resultado se obtuvo la identificación de las herramientas TIC que los estudiantes con discapacidad utilizan en su proceso de enseñanza-aprendizaje.

Palabras Claves: TIC; inclusión educativa; aprendizaje de estudiantes con discapacidad; educación inclusiva; educación superior

\begin{abstract}
University educational inclusion is a subject that has been little investigated in our country. Many variables affect the full educational inclusion of students with disabilities at a higher level. The objective of this article is to identify the ICT that the students of different careers of the Salesian Polytechnic University of Guayaquil Headquarters use to favor their inclusion, as well as the percentage of use of the ICTs offered by the UPS Support Center for Inclusion. Through bibliographic review and documentary analysis, interviews with administrative, technical and teaching staff of the university, as well as surveys applied to students with disabilities, it was possible to obtain relevant information about the ICTs used in the teaching process inclusive learning. As a result, the identification of the ICT tools that students with disabilities use in their teaching-learning process.

Key words: educational inclusion; learning students with disabilities; inclusive education; higher education
\end{abstract}




\section{Introducción}

Más de mil millones de personas viven en todo el mundo con alguna forma de discapacidad; de ellas, alrededor del 15\%, casi 200 millones experimentan dificultades considerables en su funcionamiento. (Organización Mundial de la Salud [OMS], 2011). Según el estudio del (Consejo Nacional para la Igualdad de Discapacidades [CONADIS], 2015), la atención al tema de la discapacidad en el Ecuador se inicia en las décadas del 40 y 60 con la puesta en marcha de escuelas de educación especial. Más tarde, en los años 70, en el marco del Boom Petrolero, el sector público incursiona en el desarrollo de acciones para atender a la población con discapacidad a través de programas asistenciales en educación, salud y bienestar social, fundamentalmente a través de la consolidación de servicios públicos, pero también con el reconocimiento y apoyo a la constitución de consejos como el Consejo Nacional de Rehabilitación Profesional (CONAREP) creado en el año 1973, hoy en día reconocido como CONADIS.

En el Ecuador existen 453.956 personas con diversos tipos de discapacidades, dentro de esta cifra se tiene en cuenta las siguientes: Discapacidad Física 211.997 personas (46,70\%); Discapacidad Intelectual 101.505 personas (22,36\%); Discapacidad Auditiva 64.098 personas (14,12\%); Discapacidad Visual 53.794 personas (11,85\%); Discapacidad Psicosocial 22.562 personas $(4,97 \%)$; se consideran en esta información la diversidad de género, edad y grado de discapacidad (CONADIS, 2018), tal como se observa en las Figuras 1 - 4.

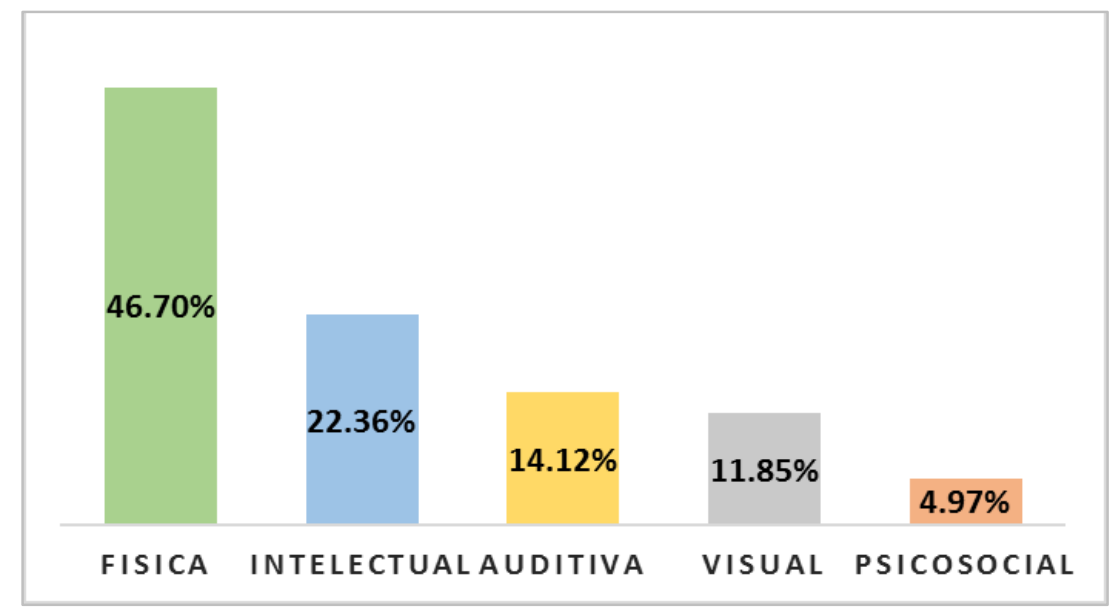

Figura 1. PcD por Tipos de Discapacidad Fuente: Ministerio de Salud Pública. Diciembre 2018 Elaborado por: Consejo Nacional para la Igualdad de Discapacidades CONADIS 


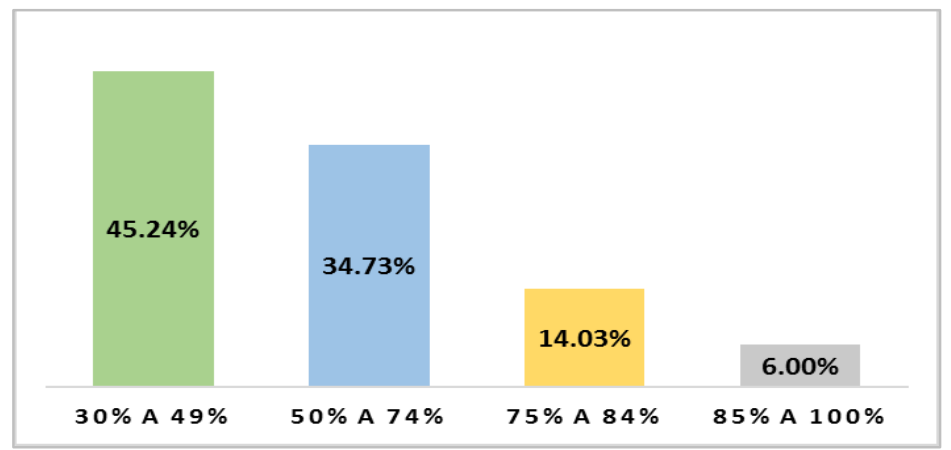

Figura 2. PcD acorde a Grados de Discapacidad

Fuente: Ministerio de Salud Pública. Diciembre 2018

Elaborado por: Consejo Nacional para la Igualdad de Discapacidades CONADIS

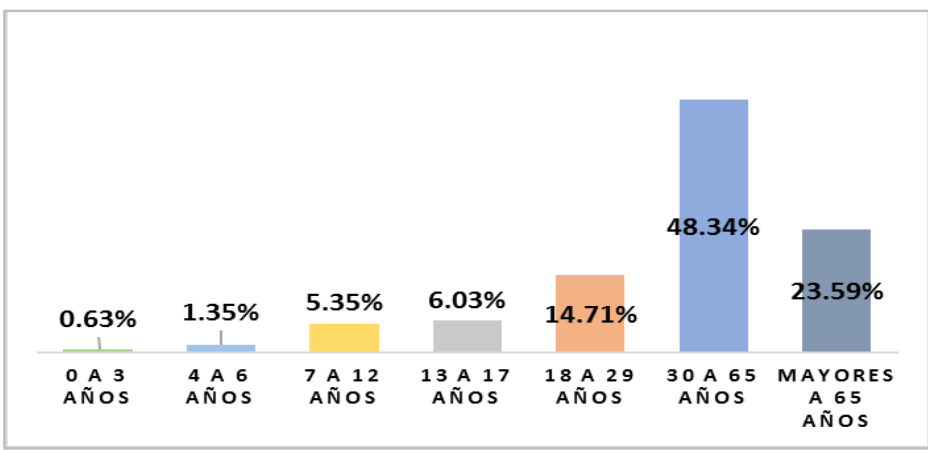

Figura 3. PcD por Grupo Etario

Fuente: Ministerio de Salud Pública. Diciembre 2018

Elaborado por: Consejo Nacional para la Igualdad de

Discapacidades CONADIS

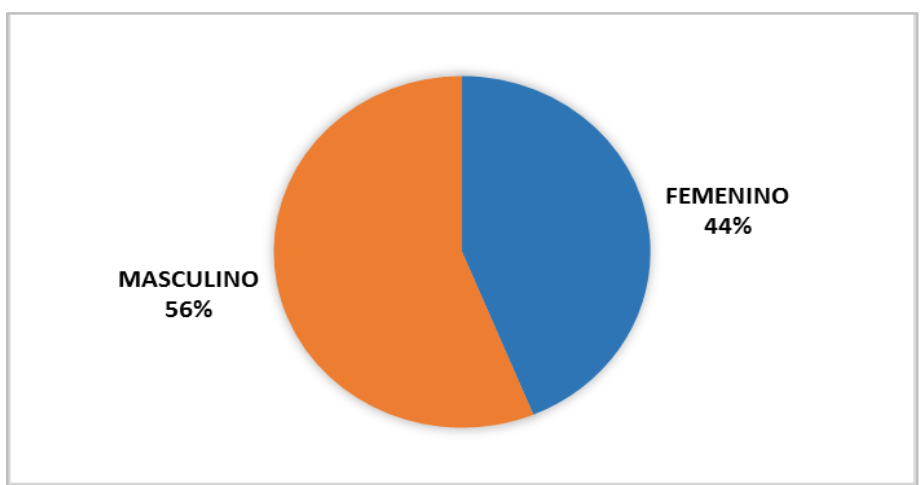

Figura 4. PcD según Género

Fuente: Ministerio de Salud Pública. Diciembre 2018

Elaborado por: Consejo Nacional para la Igualdad de

Discapacidades CONADIS

Como se aprecia a nivel nacional, existe un mayor porcentaje de personas con discapacidades físicas. El mayor porcentaje por nivel de discapacidad está comprendido en el rango del 30 al $49 \%$, las personas con discapacidad en mayor cantidad son del género masculino y las edades en que estas discapacidades están presentes están comprendidas en el rango de 30 a 
65 años. En la ciudad de Guayaquil, el comportamiento es muy similar al presentado a nivel nacional.

Se muestra como datos más relevantes que el 14,71\%, de personas con discapacidad corresponden a las edades comprendidas en el rango entre 18 a 29 años, edades en las que deberían cursar la educación superior, es decir hablamos de 67.000 jóvenes aproximadamente que deberían estar estudiando en las Universidades del país.

Por Ley, la Constitución de la República del Ecuador, artículo 47 establece que, "E1 Estado garantizará políticas de prevención de las discapacidades y, de manera conjunta con la sociedad y la familia, procurará la equiparación de oportunidades para las personas con discapacidad y su integración social, y se les reconoce el derecho a una educación que desarrolle sus potencialidades y habilidades para su integración y participación en igualdad de condiciones." (República del Ecuador, 2008, p.25). De allí que acorde a la Ley los centros de educación superior deberían brindar un entorno educativo inclusivo para los jóvenes estudiantes universitarios en el país.

Sin embargo, aún en la actualidad existen diversos factores que afectan la inclusión de estudiantes con discapacidad en los centros de educación superior, tales como, por ejemplo:

- Centros de estudios universitarios que no cuentan con infraestructura adecuada.

- Inexistencia de TIC (Tecnología de Información y Comunicación) y de recursos didácticos acorde a las necesidades particulares de cada estudiante.

- Inexistencia de productos adaptados para favorecer la inclusión educativa.

- Falta de adaptabilidad en aulas y laboratorios.

Este artículo investiga las TIC que usan los estudiantes con discapacidad, las herramientas de hardware y software con que cuenta la Universidad Politécnica Salesiana Sede Guayaquil para favorecer la inclusión de estudiantes con discapacidad, a través del Centro de Inclusión, así como el porcentaje de uso de dichas herramientas por parte de los estudiantes con discapacidad.

\section{Marco Teórico}

\section{Discapacidad}

El tema de la discapacidad se refiere a aquellas personas que presentan una disminución de sus funciones que puede ser: física, psíquica, sensorial o trastornos graves de personalidad o de conducta (OMS, 2001).

En cuanto a la clasificación de los tipos de discapacidades en la Figura 5 se presenta lo que el CONADIS, ha clasificado como discapacidad en cada grupo (CONADIS, Guía sobre Discapacidades, 2013, p.15). 


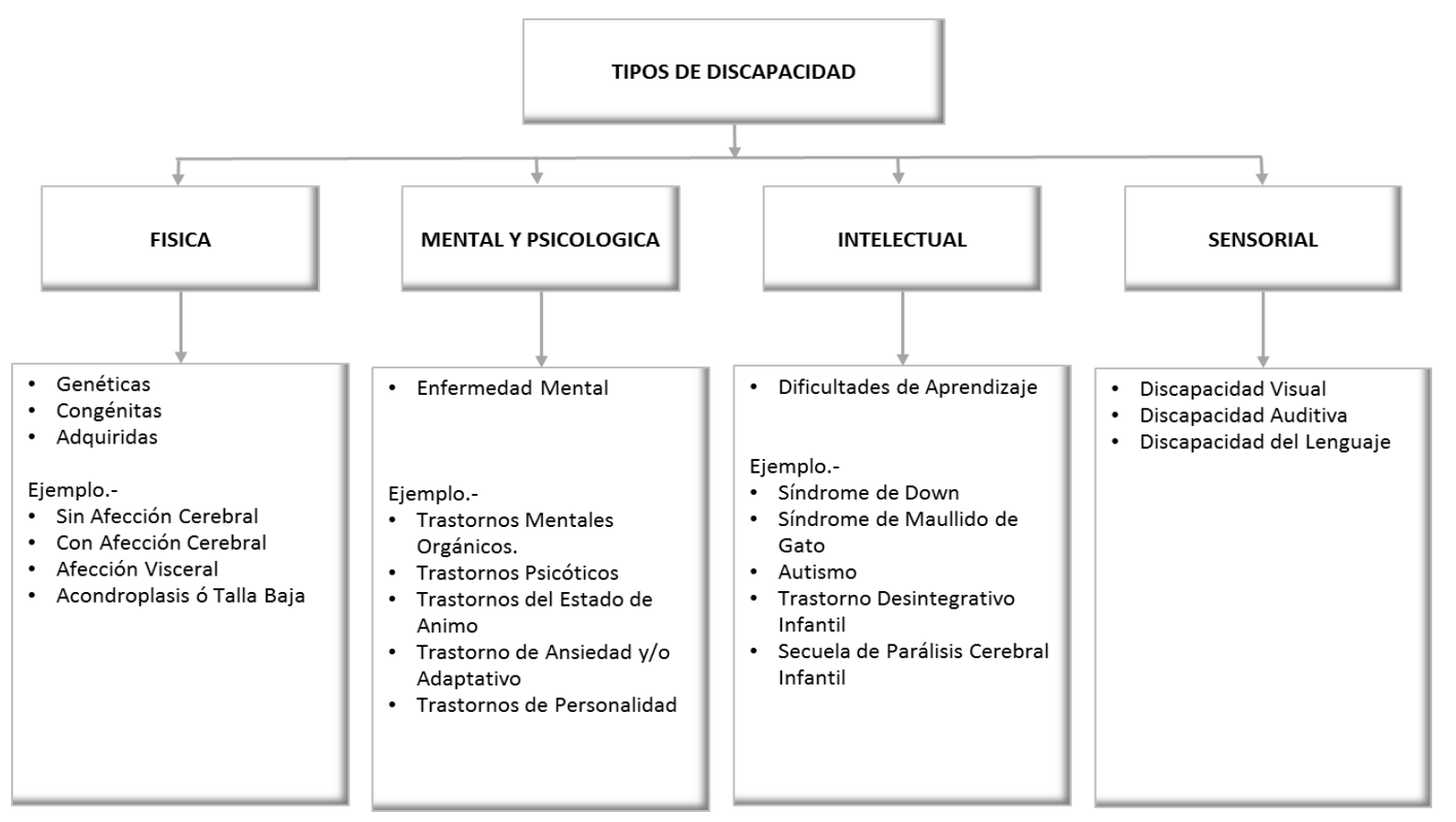

Figura 5. Clasificación de las discapacidades

Fuente: Guía sobre Discapacidades 2013

Elaborado por: Consejo Nacional para la Igualdad de Discapacidades CONADIS

En este proceso investigativo se ha encontrado que las clasificaciones de discapacidad varían de un país a otro. En este estudio se hace referencia a los tipos de discapacidades que el CONADIS describe como tales en sus informes estadísticos.

\section{Inclusión Educativa}

La educación inclusiva es considerada como un medio eficaz para educar a todos, independientemente de sus diferencias o barreras individuales o sociales (UNESCO, 2015).

El término inclusión no solo considera la integración de estudiantes en aulas, esto es insertarlos como elementos dentro del proceso enseñanza aprendizaje, la labor va más allá puesto que la educación inclusiva se debe centrar en apoyar las cualidades y necesidades de todos los estudiantes, para que se sientan bienvenidos, seguros y alcancen el éxito. (Arnáiz, 1996, p.5).

La inclusión de las personas con discapacidad en el ámbito educativo tiene especial importancia cuando se trata de hacer realidad la integración social de cualquier país, y en ese logro, la educación superior juega un papel importante (Aquino, García e Izquierdo, 2012). Por ello la inclusión educativa parecería ser un tema pendiente en algunos países, en especial en el Ecuador y ello no depende únicamente del gobierno sino también de las oportunidades que las universidades brinden a los estudiantes con discapacidad para ofrecerles una real inclusión educativa. En este sentido los elementos del proceso enseñanza aprendizaje que contribuyen a ello deben ser analizados. Cabe citar los trabajos de Arnáiz (1996 y 2000), Imbernón (1997), Egea y Sarabia (2004), Aquino, García e Izquierdo (2012), Farfán y Leyva (2015), García, Maya, 
Montalván, Pernas, Bert y Juárez (2016), Cabrera (2016) y Luque (2017), entre otros, quienes desde diferentes perspectivas analizan la inclusión educativa. Diversos enfoques y elementos del proceso inclusivo deben ser considerados, destacando no sólo lo que el estudiante con discapacidad debe hacer sino también la participación activa del docente en dicho proceso de educación inclusiva, pero éstos no son los únicos elementos que intervienen en el proceso enseñanza aprendizaje, también están las TIC.

\section{Tecnologías y personas con discapacidad}

Las TIC sirven para desarrollar nuevas formas de aprendizaje, innovaciones pedagógicas, cambios de organización, procesos de comunicación, así como romper con la acción formativa tradicional. No obstante, a todos sus beneficios algunos autores han señalado que las formas de diseñar las TIC pueden en ocasiones impedir el acceso a las mismas de sujetos con algunas limitaciones físicas, cognitivas o culturales, por lo que su diseño debe estar acorde al personal al que va dirigido. (Cabero et al., 2007); (Córdoba et al., 2012) y (Toledo, 2013), de aquí la importancia de que las TIC incluyan conceptos de diseño universal por ello en la actualidad muchas de estas herramientas han incorporado aspectos de accesibilidad para todos.

Verdecia et al., 2015, aseguran que el desarrollo de las TIC legitima a nivel global un proceso cultural que es el resultado de los avances científico-técnicos de la humanidad en diferentes contextos históricos y sociales y constituye la expresión de un nuevo espacio social donde convergen diversos intereses y experiencias y que al decir de Enríquez, (2014), dinamizan una práctica cultural donde se inscribe la vida de muchas personas y, con ello, sus oportunidades y limitaciones al momento de aprender. Este artículo investiga la importancia de las TIC en el proceso enseñanza aprendizaje por ello es preciso hacer referencia al concepto de las TIC: "Una de las disciplinas socialmente significativas que, mediante diversas actividades, responde a las demandas individuales o colectivas que presenta la sociedad" (Fallas y Trejos, 2013, p.4). Ello induce a pensar que las TIC deben ser canalizadas de forma individual acorde a las necesidades de cada alumno con discapacidad en el aula.

Las TIC son entonces utilizadas tanto por docentes como por estudiantes en los entornos universitarios con mayor incidencia que en otros niveles educativos y eso se destaca en investigaciones previas como la de Wagh que indica que "el uso de las tecnologías como apoyo al aprendizaje de los estudiantes es usada por los docentes en clases como los demuestran algunos estudios" (Wagh, 2012, p.2195).

\section{Metodología}

La modalidad de la presente investigación es bibliográfica, documental, descriptiva y de evaluación cuali-cuantitativa. Se empleó la búsqueda través de internet, en bases indexadas, para consultar aspectos relacionados a la temática propuesta en este artículo.

Las técnicas utilizadas en este artículo fueron entrevista y encuesta, así como el análisis documental y la observación. 
La entrevista fue dirigida a personal administrativo, técnico y docente, que se encuentra en contacto con los estudiantes tales como recepcionistas, secretarias, bienestar estudiantil docente y personal de sistemas de la Universidad Politécnica Salesiana Sede Guayaquil. A nivel docente se relevó información de 12 docentes, uno por cada carrera de pregrado existentes en la UPS al 2018.

Se diseñó un cuestionario dirigido a estudiantes con discapacidad para conocer las TIC que ellos utilizan en su proceso de enseñanza aprendizaje. De los 104 estudiantes con discapacidad registrados en el 2018 , solo el $50 \%$ respondieron a la encuesta en línea enviada para el efecto, dentro de este proceso investigativo.

Se solicitaron los datos oficiales de la población estudiantil con discapacidad registrada en la UPS, así como se revisó la documentación a manera de registros que el departamento que atiende y da soporte tecnológico y de apoyo a los estudiantes con discapacidad mantiene, en especial de los servicios de préstamo de TIC a estudiantes con discapacidad.

Se realizaron visitas al centro de apoyo y se aplicó la técnica de observación para constatar la información relevada en entrevistas y cuestionarios.

\section{Resultados}

Luego de las entrevistas realizadas al personal administrativo de la UPS con la finalidad de conocer las TIC con que la UPS cuenta para los estudiantes con discapacidad se identificó que en la Universidad Politécnica Salesiana Sede Guayaquil existe el Centro de Apoyo para la Inclusión (CAI), el cual brinda asistencia especializada a estudiantes con discapacidad y da soporte durante toda su vida estudiantil, un acompañamiento especializado para que los estudiantes con discapacidad puedan estudiar en igualdad de condiciones con el apoyo de recursos, equipos tecnológicos de hardware y software disponibles en dicho centro. El personal administrativo nos indicó que allí se encuentran las TIC para personas con discapacidad.

Este centro de apoyo fue creado desde el 2013, a través de un proyecto de investigación que analizó el tema de la inclusión educativa de estudiantes con discapacidad y determinó la necesidad de crearlo para brindar una serie de servicios y TIC a los estudiantes con discapacidad de la UPS, así como a la sociedad.

Esta información fue corroborada en el sitio web de los UPS, cuyo centro es referido dentro del espacio físico de la biblioteca ubicado en el Bloque F, 2do piso dentro de las mismas instalaciones de la Universidad Politécnica Salesiana Sede Guayaquil, se visitó el centro y se aplicó la técnica de la observación, allí se encuentran registros y documentos que permiten evidenciar, el acceso tanto de estudiantes como a docentes de todos los servicios que este centro brinda, además se constató las TIC disponibles que se mantienen en exhibición en perchas, modulares y/o vitrinas.

En el relevamiento de información se nos explicó que el CAI, cuenta con un inventario de las TIC existentes, el cual nos fue facilitado con fines de investigación. En este centro se pudo obtener la información estadística de los estudiantes con discapacidad de los periodos \# 50, \# 51, 
cuyo total es de 104 estudiantes en el 2018, pertenecientes a las diversas carreras que la UPS ofrece. A través del análisis documental y la tabulación de resultados se encontró que las principales discapacidades que se presentan en los estudiantes registrados son las físicas, seguidas de las visuales (Figura 6), el grupo etario se encuentra comprendido en el rango de 21 a 24 años de edad, con mayor porcentaje del sexo masculino (Figura 7), y las carreras que involucran un mayor número de estudiantes con discapacidad son las de Computación, Comunicación Social, Administración de Empresas, y Contabilidad y Auditoría (Figura 8).

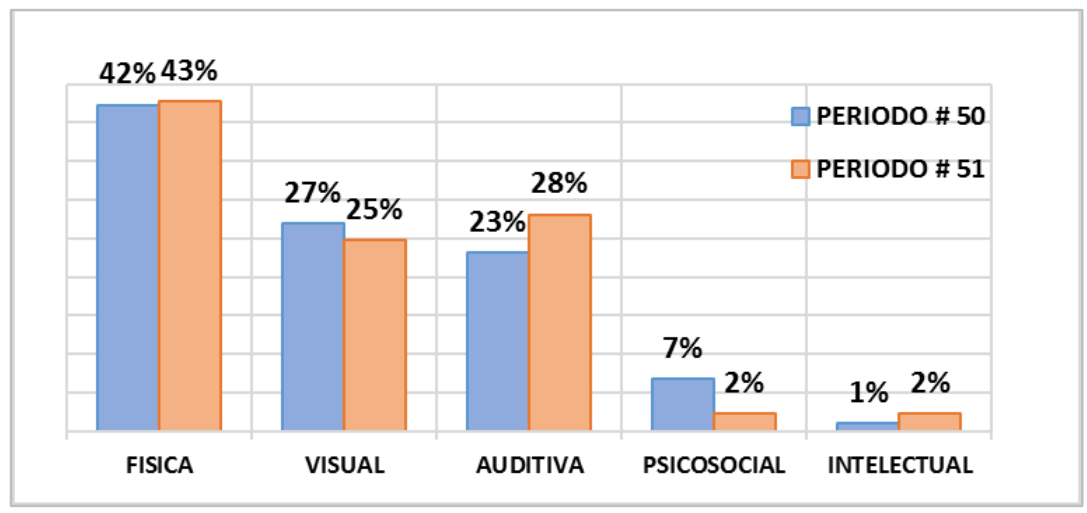

Figura 6. Población de estudiantes con discapacidad de la UPS Sede Guayaquil.

Fuente: Dpto. Bienestar Estudiantil UPS Sede Guayaquil 2018

Elaborado por: Autores

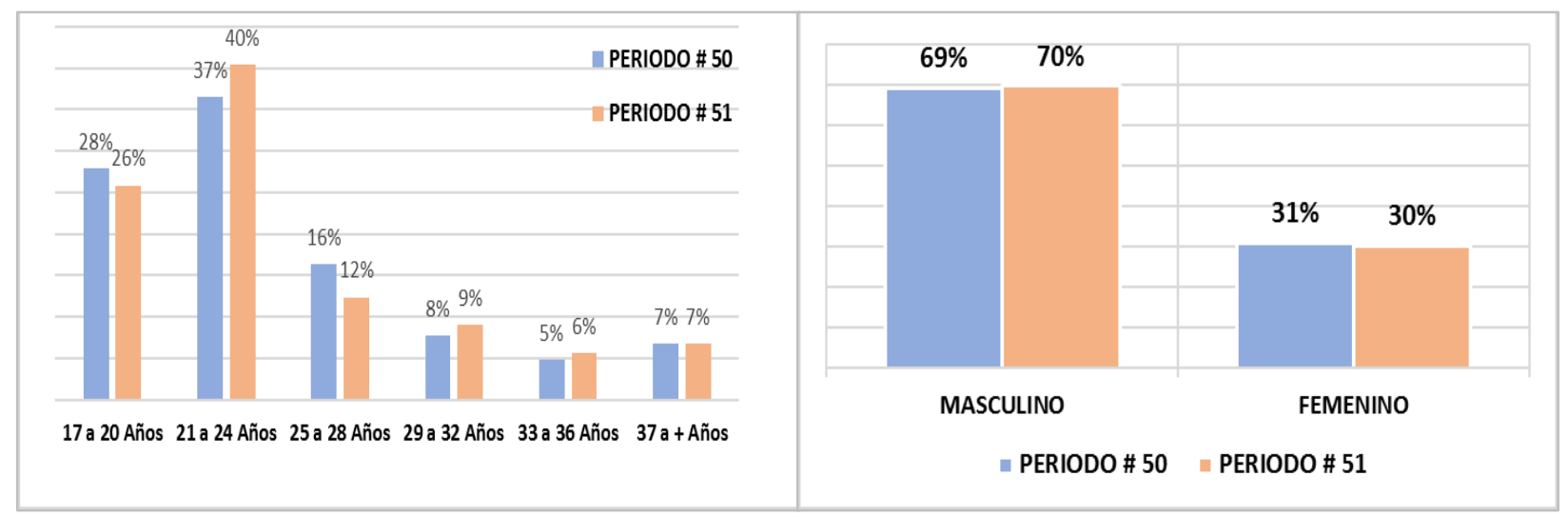

Figura 7. Clasificación en Grupo Etario y Género de los estudiantes de la UPS Sede Guayaquil. Fuente: Dpto. Bienestar Estudiantil UPS Sede Guayaquil 2018

Elaborado por: Autores 


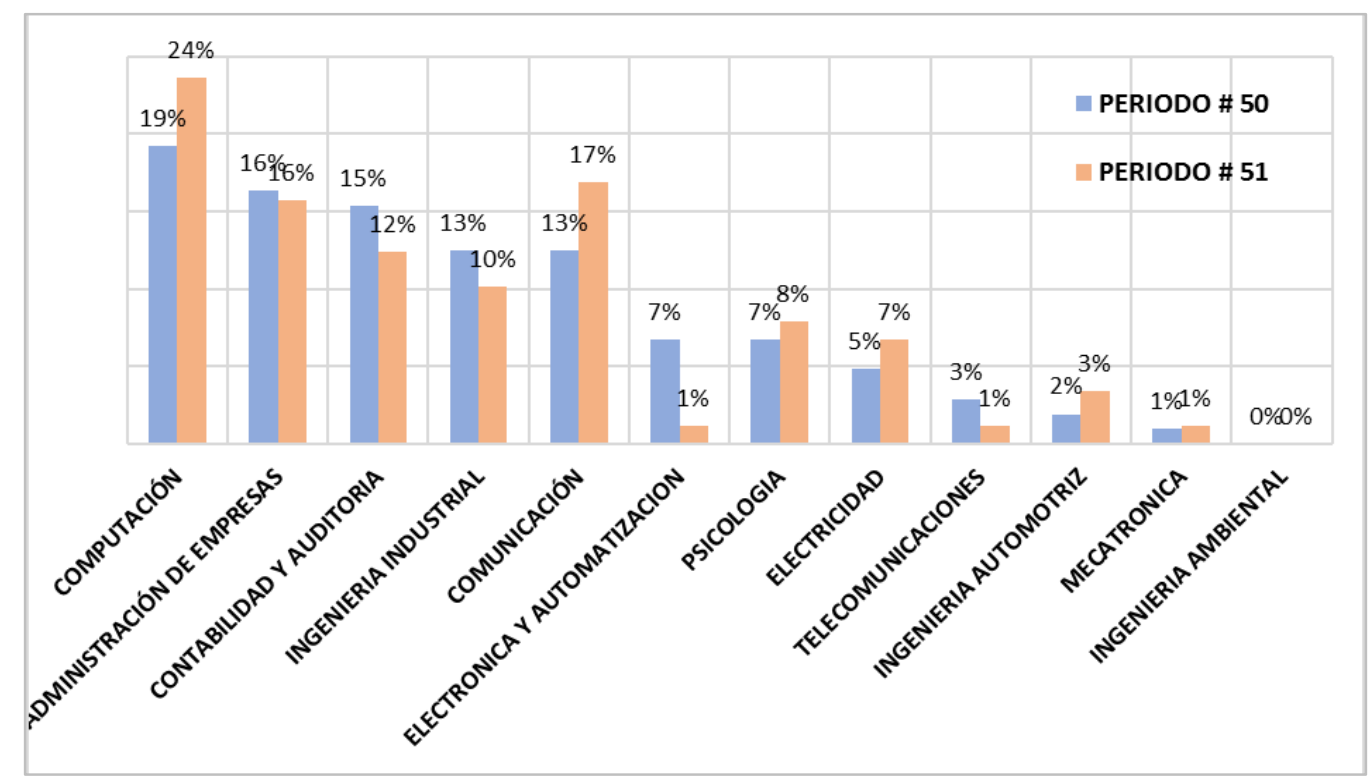

Figura 8. Distribución de los estudiantes con discapacidad de la UPS Sede Guayaquil por carreras.

Fuente: Dpto. Bienestar Estudiantil UPS Sede Guayaquil 2018

Elaborado por: Autores

En la Figura 9, se observan las TIC para la inclusión de estudiantes con discapacidades existentes en el CAI, disponibles tanto para los docentes como para los estudiantes de la sede y otras sedes e inclusive para la sociedad, todas ellas orientadas a fomentar el proceso educativo inclusive en instituciones de Educación General Básica (EGB), bachillerato y universidades. 


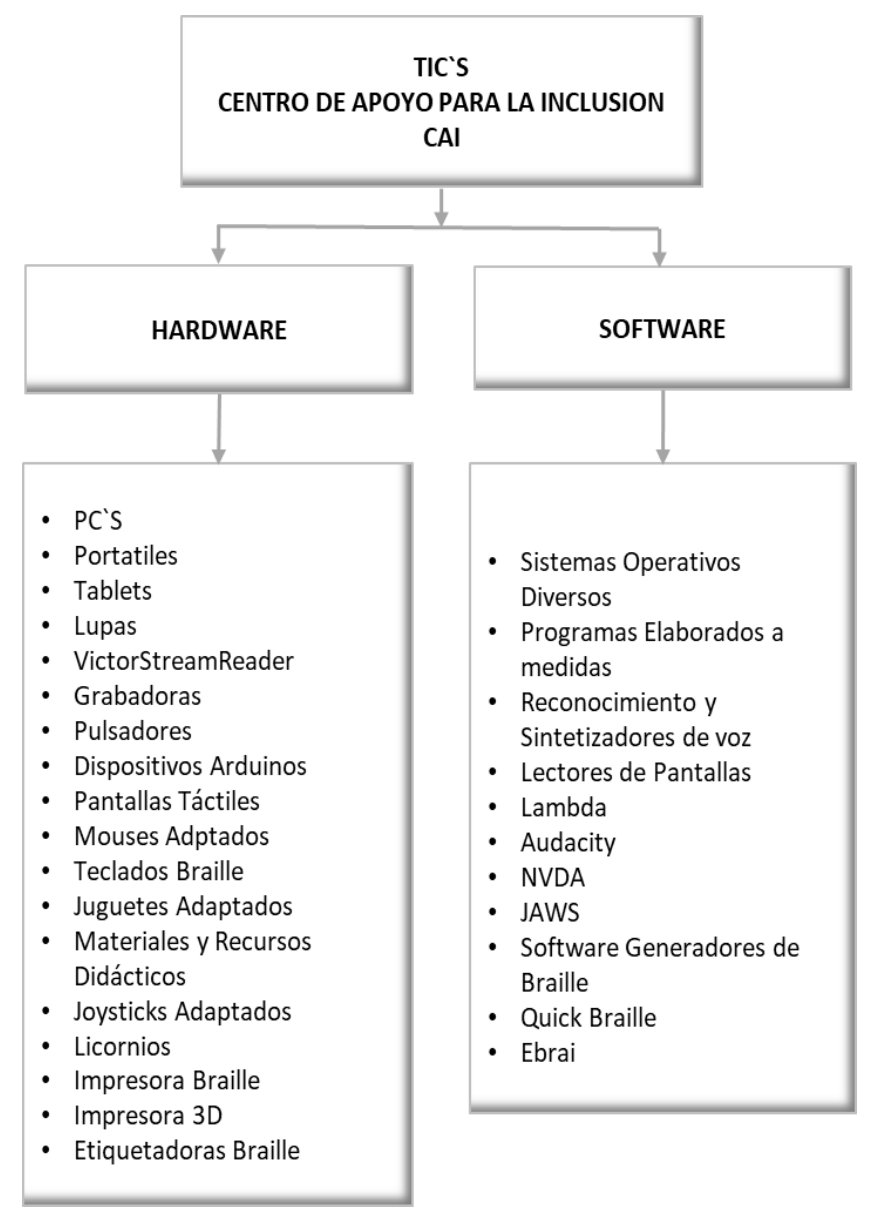

Figura 9. Principales TIC con que cuenta el CAI para el aprendizaje inclusivo.

Fuente: Dpto. Centro de Apoyo para la Inclusión UPS

Sede Guayaquil 2018

Elaborado por: Autores

Las Tablas 1 y 2, presentan las herramientas en hardware y software, con que cuenta el CAI de la Universidad Politécnica Salesiana Sede Guayaquil, agrupadas por tipo. 
Tabla 1. Herramientas Hardware del CAI.

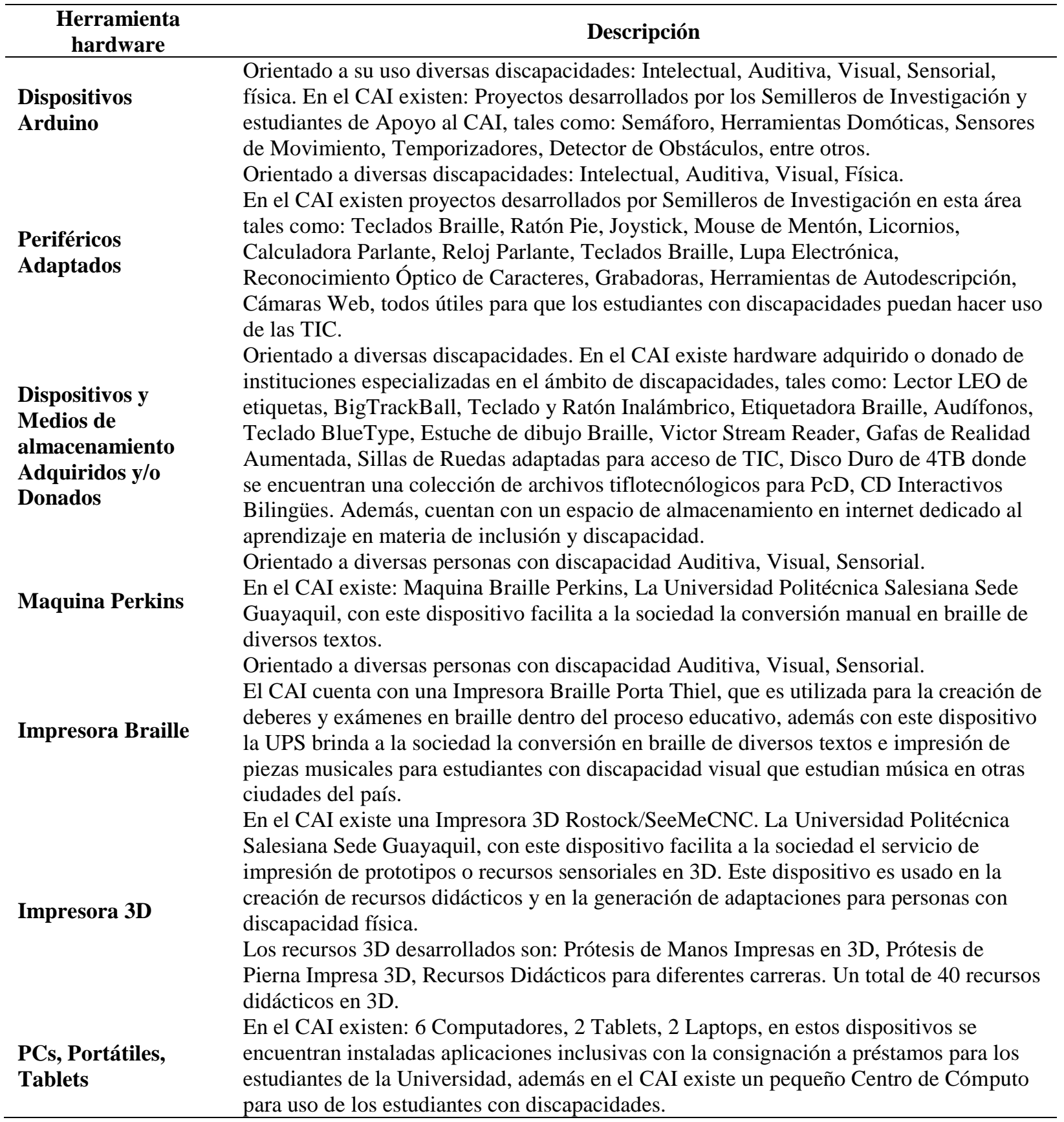

Fuente: Elaboración propia. 
Tabla 2.

Herramientas Software del CAI.

\begin{tabular}{cc}
$\begin{array}{c}\text { Herramienta } \\
\text { software }\end{array}$ & Descripción \\
\hline
\end{tabular}

Orientado a diversas personas con discapacidad Auditiva, Visual, Sensorial.

NVDA Non Visual Desktop Access, es un lector de pantalla de código abierto gratuito con salida de síntesis de voz e interfaz braille, es usado para el sistema operativo Windows. Esta

Lectores de aplicación se encuentra instaladas en los laboratorios de cómputo de la UPS Sede Guayaquil,

Pantalla disponibles para su uso en clases prácticas.

JAWS Job Access With Speech, es un lector de pantalla para personas ciegas o con visión reducida. Su finalidad es hacer que ordenadores personales que funcionan con Microsoft Windows sean más accesibles para personas con alguna discapacidad relacionada con la visión. Se encuentra instalada en el CAI y en las portátiles que presta.

Orientado a diversas personas con discapacidad Visual.

TIFLOBUNTU es una distribución Linux, basada en Ubuntu, permite personalizar al computador para hacer más fácil el acceso al sistema operativo a personas que sufren alguna discapacidad visual.

VINUX es una distribución de Linux, basada en Ubuntu, especialmente diseñada para usuarios con discapacidad visual, con herramientas para convertir el texto en voz (al estilo JAWS), lupas dinámicas para visualizar mejor el contenido de la pantalla, o soporte para teclados

Sistemas Operativos

Utilitarios

\section{braille USB.}

WINDOWS es un conjunto de programas que posibilita la administración de los recursos de una computadora, este tipo de sistemas empieza a trabajar cuando se enciende el equipo para gestionar el hardware a partir desde los niveles más básicos. En el Centro de Apoyo para la Inclusión, existen las diversas versiones tales como: Windows XP, Windows 8, Windows 10, con la finalidad de utilizar los diferentes aplicativos por compatibilidad de software.

ANDROID es el sistema operativo móvil desarrollado por Google, basado en el Kernel de Linux y otros softwares de código abierto. Fue diseñado para dispositivos móviles con pantalla táctil, como teléfonos inteligentes, tabletas, relojes inteligentes, automóviles y televisores. Orientado a diversas personas con discapacidad Visual. Física e Intelectual.

LAMBDA es un editor matemático que permite que un alumno ciego, el profesor y el resto de los alumnos interactúen en esta asignatura de forma eficaz. La signografía matemática tradicional no puede ser percibida tal cual, por una persona ciega, precisa ser transcrita a Braille, de esto también se encarga el programa. En sentido contrario, el alumno ciego debe escribir las matemáticas siguiendo una signografía especial y que no sería fácilmente interpretable para un profesor o el resto de los alumnos.

QUICK BRAILLE es un procesador de textos que permite transcribir textos a formato Braille, para lo cual es posible teclear directamente el texto o abrir un documento en formato RTF o TXT. Esta aplicación es de uso frecuente por parte de los profesionales que se encargan de la transcripción de documentos a Braille en el CAI.

EBRAI es un software para la edición de textos en sistema braille, que está pensado tanto para usuarios particulares, que desean convertir textos en tinta fácilmente a braille, de materiales educativos para los estudiantes de la UPS y para la sociedad.

AUDACITY es una aplicación informática multiplataforma libre, que se puede usar para grabación y edición de audio, distribuido bajo la licencia, es el editor de audio más difundido en los sistemas y es usado para convertir archivos de texto a voz.

EVIACAM es una herramienta de ayuda a $\mathrm{PcD}$, que se engloba dentro de las llamadas tecnologías de apoyo. Éstas pueden dividirse en cinco grupos: sistemas alternativos y aumentativos de acceso a la información, sistemas de acceso, sistemas alternativos y aumentativos de comunicación, sistemas de movilidad y sistema de control de entornos. Es un sistema accesible, lo que quiere decir que sirve para permitir a las personas con discapacidad física o sensorial utilizar el ordenador. Se usa en el CAI y se instala según requerimiento.

ARABOARD es un conjunto de herramientas diseñadas para la comunicación alternativa y aumentativa, cuya finalidad es facilitar la comunicación funcional, mediante el uso de 
imágenes y pictogramas, a personas que presentan algún tipo de dificultad en este ámbito. EL CAI a través de los semilleros crea aplicaciones a medida para las personas que los soliciten. LIBRE OFFICE es un paquete de software de oficina libre diseñada para ser compatible con los principales paquetes ofimáticos, incluido Microsoft Office, aunque algunas características de diseño y atributos de formato son diferentes, siendo una herramienta accesible.

MICROSOFT OFFICE es un paquete de programas informáticos para oficina, con licencia y cuenta con un conjunto de aplicaciones que son usadas en las actividades educativas.

Orientado a diversas personas con discapacidad Auditiva, Visual, Intelectual, Sensorial. En el CAI existen muchos proyectos desarrollados por Semilleros de Investigación y estudiantes, tales como:

Aplicativos INFORMATIC GAMES Aplicación que permite participar en diferentes juegos para Móviles potenciar las habilidades sensoriales y motrices.

AGENDA ASISTENTE Aplicación que permitirá saber que opción está usando, tendrá una asistente, agenda y reloj parlante.

Existiendo entre un total de 40 aplicaciones desarrolladas para atender diversas necesidades en el ámbito de educación inclusiva.

SIMPLIFY3D Traduce los modelos 3D en instrucciones para poder ser presentados en la Aplicaciones impresora 3D. Es usada en la creación de recursos didácticos en materia educativa.

$3 D$ ULTIMAKER CURA Prepara un modelo para la impresión 3D. Es utilizado en el diseño, prototipado e impresión en 3D.

Permite el acceso a la Web, interpretando la información de distintos tipos de archivos y sitios web para que estos puedan ser visualizados, permite importar y agregar plugin de accesibilidad

Navegadores entre otras funcionalidades más. Las usadas son: GOOGLE CHROME, INTERNET

EXPLORER, MOZILLA FIREFOX. La Universidad Politécnica Salesiana Sede Guayaquil, con estos aplicativos facilita la interacción con internet a las personas con distintas discapacidades, permitiendo la utilización de sitio web interactivos e inclusivos.

Fuente: Elaboración propia.

Las TIC de la Tablas 1 y 2 están a disposición tanto de estudiantes, así como de docentes para Educación General Básica (EGB) y Bachillerato General Unificado (BGU) que forman parte de la Red Académica de Apoyo e Investigación en Tecnologías Inclusivas (RAITI).

Adicionalmente, al analizar los registros del Centro de Apoyo para la Inclusión, se encontró el porcentaje de uso de las herramientas del CAI, de los periodos 50 - 51 (Ver Figura $10)$.

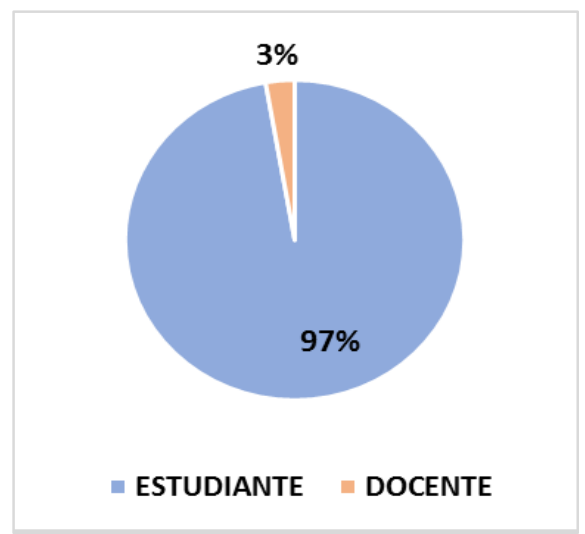

Figura 10. Porcentaje de uso de las herramientas del CAI por tipos de Usuarios.

Fuente: Dpto. Centro de Apoyo para la Inclusión UPS

Sede Guayaquil 2018

Elaborado por: Autores 
En la Figura 10 podemos encontrar que el porcentaje de uso de las herramientas TIC del CAI es del $97 \%$ y el 3\% de los docentes, esto acorde a los registros de préstamos que el centro mantiene, además, las carreras que utilizan las TIC del CAI se presentan en la Figura 11, destacándose la carrera de computación.

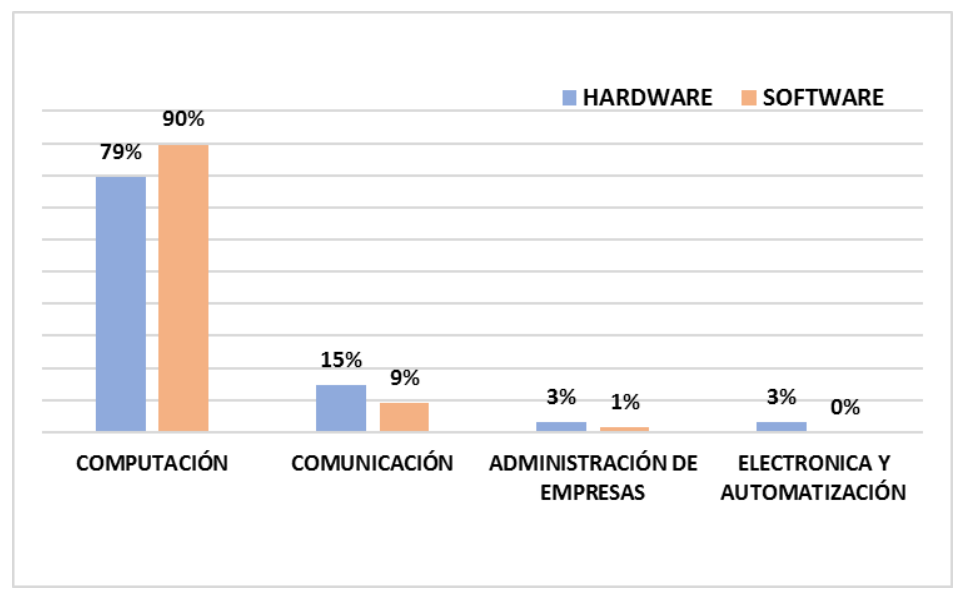

Figura 11. Carreras que emplean con mayor frecuencia el hardware y el software del CAI.

Fuente: Dpto. Centro de Apoyo para la Inclusión UPS Sede Guayaquil 2018

Elaborado por: Autores

También se encontró que los equipos y dispositivos tanto de hardware como de software más utilizados por los estudiantes, en el proceso educativo son: laptops, punzones braille, teclado braille, disco duro (Tifloteca), en materia de hardware.

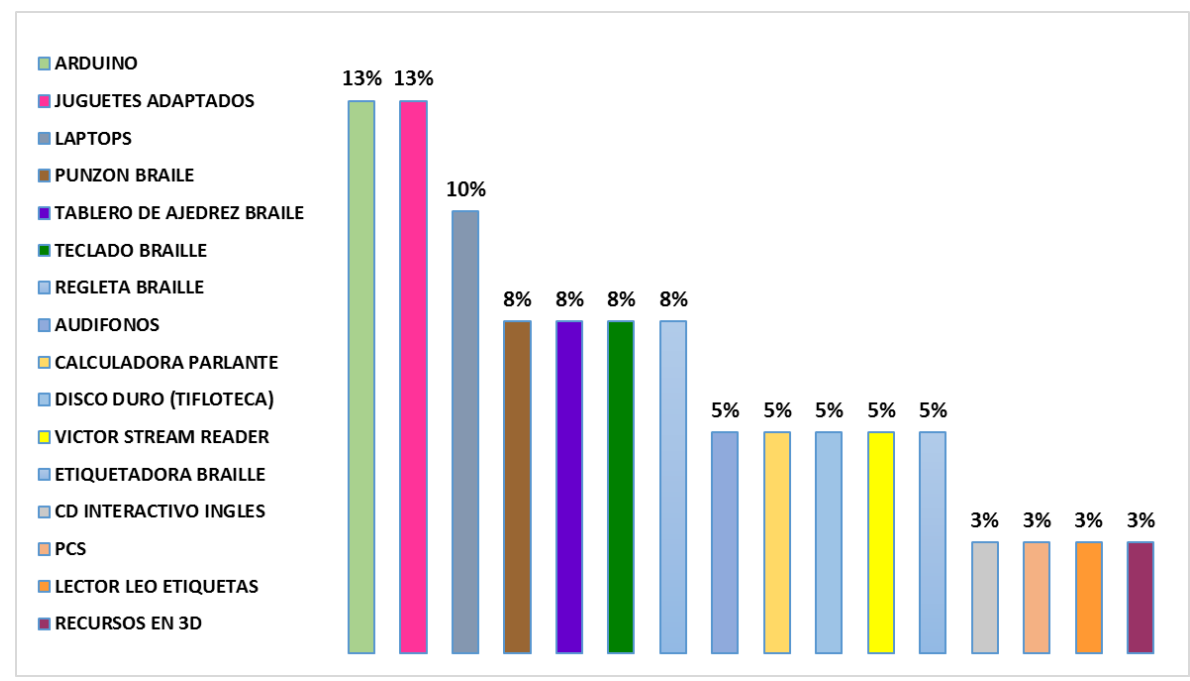

Figura 12. Herramientas Hardware más utilizados por los estudiantes en el CAI. Fuente: Dpto. Centro de Apoyo para la Inclusión UPS Sede Guayaquil 2018 Elaborado por: Autores 
Como se observa en la figura 12 algunas de las herramientas que se emplean con más frecuencia por parte de estudiantes son Arduino y Juguetes Adaptados, herramientas usadas para favorecer la inclusión educativa en otras entidades de la sociedad. Los estudiantes utilizan estas herramientas para su trabajo en proyectos de vinculación con la sociedad donde favorecen el uso de las TIC adaptativas a instituciones educativas de EGB y de entidades como asociaciones o federaciones de personas con discapacidad. Si bien esto no forma parte de este estudio, es importante destacar el destino de dichos préstamos.

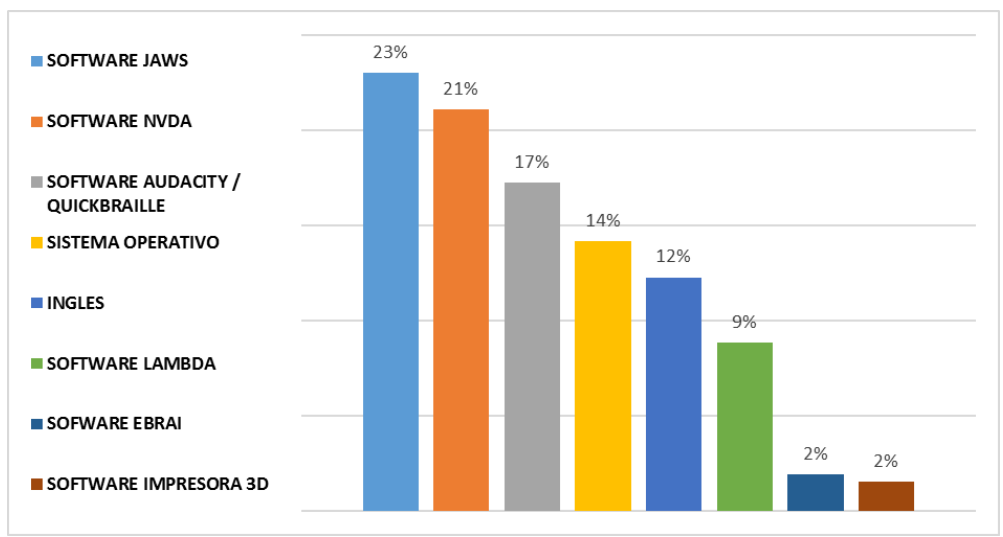

Figura 13. Herramientas Software más utilizados por los estudiantes en el CAI.

Fuente: Dpto. Centro de Apoyo para la Inclusión UPS Sede Guayaquil 2018

Elaborado por: Autores

En la figura 13 se destaca el uso de lectores de pantalla como JAWS y NVDA por parte de los estudiantes con discapacidad visual. Estas herramientas también se encuentran instaladas en los laboratorios de la UPS para facilitar la inclusión de estudiantes en esas instalaciones, lo que pudimos constatar en la visita efectuada a los mismos.

En las entrevistas realizadas a docentes seleccionados, acorde a muestreo dirigido, se pudo encontrar que las TIC que usan los docentes son: Sistemas operativos propietarios y libres, Máquinas virtuales, laboratorios especializados y de computación, entorno virtual de aprendizaje denominado AVAC (Ambiente Virtual de Aprendizaje Cooperativo), simuladores, software gratuito y comercial disponible acorde al aprendizaje en cada carrera, herramientas de la nube gratuitas y pagadas, foros, wikis, blogs institucionales, internet, YouTube, videos propios, herramientas ofimáticas de Windows y LibreOffice, entre otros, además de contar con hardware como: computadoras, proyectores, tablets, servidores, redes de computadoras, almacenamientos en la nube institucional y particulares, contratados por cada docente.

La encuesta aplicada a estudiantes con discapacidad dio como resultado que, en su proceso de inclusión educativa, usan en un 100\% las TIC, el 70\% indicó conocer la existencia de las TIC del CAI, 30\% indicó hacer uso del laboratorio o centro de cómputo del CAI, mientras que un $100 \%$ indicó usar los laboratorios de la UPS y el AVAC.

\section{Conclusiones}

Como resultado se obtuvo la identificación de las herramientas TIC que la UPS ofrece a los estudiantes con discapacidad a través del CAI, así como el nivel de uso de estas herramientas 
por parte de los estudiantes de diferentes carreras, alcanzando un porcentaje de solicitudes de préstamo de hardware y software de un $97 \%$ por parte de los estudiantes que acudieron a dicho centro, evidenciando que los estudiantes perciben a las TIC como herramientas de apoyo importantes en el proceso de enseñanza-aprendizaje.

Además, un grupo de estudiantes con discapacidad usan TIC adaptadas a sus necesidades particulares. Dichos resultados fueron corroborados mediante la triangulación de técnicas aplicadas: entrevistas, encuestas y análisis documental, destacando entre ellas las herramientas que usan las personas con discapacidad visual como: lectores de pantalla, victor stream reader y sistema braille.

Lo que confirma lo expuesto por autores, tales como, Naranjo (2017), que indico que, para el desarrollo de la inclusión educativa es necesario contar con TIC, y éstas deben ser implementadas en todas las instituciones para favorecer los procesos de comunicación e interrelación docente-estudiante con y sin discapacidad, puesto que constituyen un mecanismo para mejorar la calidad de los procesos de aprendizaje, ello se evidencia en esta investigación al encontrar que tanto docentes como estudiantes consideran importante el uso de las TIC en el proceso de enseñanza-aprendizaje destacándose coincidencias en TIC, tales como: el ambiente Virtual de Aprendizaje Cooperativo (AVAC), herramientas de la nube, herramientas ofimáticas, computadoras, portátiles, internet.

Mediante esta investigación se infiere que en las instituciones de educación superior es deseable la existencia de un departamento que favorezca el proceso de enseñanza y aprendizaje inclusivo, así como que las instituciones educativas provean a sus estudiantes de TIC adaptativas que permitan satisfacer las necesidades individuales de los estudiantes con discapacidad para su uso en aulas o laboratorios.

\section{Agradecimientos}

Al Centro de Apoyo para la Inclusión de la Universidad Politécnica Salesiana Sede Guayaquil, por facilitar información para el desarrollo de esta publicación.

Al Grupo de Investigación TICAD (Tecnologías de Información y Comunicación asociadas a discapacidad) por el apoyo brindado en el desarrollo de esta investigación.

\section{Bibliografía}

Aquino Zúñiga, S. P. \& García Martínez, V. \& Izquierdo J. (2012). La inclusión educativa de ciegos y baja visión en el nivel superior. Un estudio de caso. Universidad Jesuita de Guadalajara. Sinéctica 39. Obtenido de: http//www.sinectica.iteso.mx

Arnáiz, P. (1996). Las escuelas son para todos. Siglo Cero 27 (2), 1996, pp 25- 34.

Arnáiz, P. (2000). Hacia una educación eficaz para todos: La educación inclusiva. Obtenido de: http://www4.congreso.gob.pe/comisiones/2006/discapacidad/tematico/educacion/inclusio n.pdf

Cabero J, Córdoba M, Fernández JM. (2007). Las Tics para la igualdad. Nuevas tecnologías y atención a la diversidad. Edit. Eduforma. Sevilla. 
Cabrera B. (2016). La estrategia pedagógica como escenario de acción para el mejoramiento del desempeño profesional de los docentes de la Universidad Católica de Cuenca. Revista Cubana de Educación Superior. (1).41-50.

CONADIS. Consejo Nacional de Igualdad de Discapacidades. (2013). Guía sobre Discapacidades.

CONADIS. Consejo Nacional de Igualdad de Discapacidades. (2015). Ecuador: La discapacidad en cifras Análisis de resultado de la encuesta nacional de discapacidades.

CONADIS. Consejo Nacional de Igualdad de Discapacidades. (2018). Recuperado de: http://ecuadorinmediato.com/index.php?module=Noticias\&func=news_user_view\&id=12 6210\&umt=gobierno_anuncia_nuevo_programa_atencion_a_discapacitados.

Córdoba M, Cabero J, Soto Pérez FJ. (2012). Buenas prácticas de aplicación de las Tics para la igualdad. Edit. Eduforma. Sevilla

Enríquez S. (2014). Comunicar educación. Apuntes desde la teoría y la práctica sobre el desafío de enseñar a través de medios digitales. Documento de trabajo. Ministerio de Planificación Federal/ Inversión Pública y Servicios, Buenos Aires.

Fallas V, Trejos I. (2013). Educación en la sociedad de la información y el conocimiento. San José, Costa Rica: EU-NED

Farfán P, Leyva AL. (2015). Las tecnologías de la información y las comunicaciones en el proyecto educativo de la Universidad Politécnica Salesiana del Ecuador. Revista Cubana de Educación Superior. Número 3. 114-130

García ML, Maya GP, Pernas IA, Bert JE, Juárez V. (2016). La tutoría con enfoque inclusivo desde la universidad para estudiantes con discapacidad visual. Revista Cubana de Educación Superior. Número 3. 148-160.

Naranjo Sánchez, B. A. (2017). Elementos básicos para la inclusión educativa de estudiantes con discapacidad. Boletín Virtual - Agosto - Vol. 6 - 8.

Luque MP. (2017). La formación del docente en la educación inclusiva universitaria. Recus: Revista Electrónica Cooperación Universidad - Sociedad. Vol. 1, No. 2 (Abril - Agosto)

Organización Mundial de la Salud. (2001). Clasificación internacional del funcionamiento, de la discapacidad y de la salud. Ginebra: OMS. Organización

Organización Mundial de la Salud. (2011). Informe mundial sobre la discapacidad. Ginebra: OMS. Recuperado de https://www.who.int/disabilities/world_report/2011/es/

República del Ecuador. (20 de Octubre de 2008). Constitución de la República del Ecuador. Montecristi, Ecuador. Recuperado de https://www.asambleanacional.gob.ec/sites/default/files/documents/old/constitucion_de_ bolsillo.pdf

Toledo P. (2013). Las tecnologías de la información, la comunicación y la inclusión educativa. En Barroso J. \& Cabero J. Nuevos escenarios digitales. Edit. Pirámide. Madrid. 411-426.

UNESCO (2015). Declaración de Incheon y Marco de Acción para la realización del Objetivo de Desarrollo Sostenible. Recuperado de https://bit.ly/2ejZBWa

Verdecia E, Enríquez SC, Gargiulo SB, Ponz MJ, Scorians EE, Vernet M, Wenk N. (2015). Tecnologías de la información y las comunicaciones en educación. Logros actuales y proyección hacia el futuro. Revista Cubana de Educación Superior. Número 2. 4-17.

Yuni J, Urbano C. (2016). Técnicas para investigar: recursos metodológicos para la preparación de proyectos de investigación. Córdoba: Brujas

Wagh R. (2012) “Using Scrum for Software Engineering Class Projects” in, India, Goa: Agile, pp. 68-71, February 